\title{
Experimental Psychology, Animal
}

National Cancer Institute

\section{Source}

National Cancer Institute. Experimental Psychology, Animal. NCI Thesaurus. Code C18783.

The use of animals to study psychological traits such as math reasoning or ability to learn. 